\title{
Oscillators and operational amplifiers
}

\author{
Lindberg, Erik
}

Published in:

Proceedings of the 2005 European Conference on Circuit Theory and Design

Link to article, DOI:

10.1109/ECCTD.2005.1522982

Publication date:

2005

Document Version

Publisher's PDF, also known as Version of record

Link back to DTU Orbit

Citation (APA):

Lindberg, E. (2005). Oscillators and operational amplifiers. In Proceedings of the 2005 European Conference on Circuit Theory and Design (Vol. 2, pp. II19-II22). IEEE. https://doi.org/10.1109/ECCTD.2005.1522982

\section{General rights}

Copyright and moral rights for the publications made accessible in the public portal are retained by the authors and/or other copyright owners and it is a condition of accessing publications that users recognise and abide by the legal requirements associated with these rights.

- Users may download and print one copy of any publication from the public portal for the purpose of private study or research.

- You may not further distribute the material or use it for any profit-making activity or commercial gain

- You may freely distribute the URL identifying the publication in the public portal

If you believe that this document breaches copyright please contact us providing details, and we will remove access to the work immediately and investigate your claim. 


\title{
Oscillators and Operational Amplifiers
}

\author{
Erik Lindberg, IEEE Lifemember*
}

\begin{abstract}
A generalized approach to the design of oscillators using operational amplifiers as active elements. A piecewise-linear model of the amplifier is used so that it make sense to investigate the eigenvalues of the Jacobian of the differential equations. The characteristic equation of the general circuit is derived. The dynamic nonlinear transfer characteristic of the amplifier is investigated. Examples of negative resistance oscillators are discussed.
\end{abstract}

\section{INTRODUCTION}

When you want to design an oscillator the Barkhausen Criteria is normally used as a starting point i.e. a linear circuit with poles on the imaginary axis is designed $[1,2]$. In order to startup oscillations some parameters are changed so that the poles are in the right half of the complex frequency plane. The linear circuit becomes unstable and the signals will grow until infinity i.e. we must introduce nonlinearity in order to limit the signal amplitude.

\section{- Oscillators are nonlinear circuits.}

In the following an oscillator design procedure is described. It is based on the idea of "... . . . . . .

" [3]. By means of piecewise linear modelling of the nonlinearities the circuit is linear in time slots so that it make sense to study the eigenvalues (poles) of the Jacobian of the differential equations and see whether the signals are increasing or decreasing in amplitude in a certain time slot. If you observe that the poles are moving back and forth between the right half (RHP) and the left half (LHP) of the complex frequency plane the mechanism behind the behavior of an oscillator may be described as an act of balance between the energy you gain from the dc power supply when the poles are in RHP and the energy you loose when the poles are in LHP. The characteristic equation for a general linear circuit with a perfect amplifier with a piecewise linear transfer characteristic is derived. The dynamic transfer characteristic of a specific operational amplifier (op amp) RC4136 is studied by means of PSpice analysis.

* Ørsted•DTU Department, 348 Technical University of Denmark, DK2800 Kongens Lyngby, DENMARK, e-mail: el@oersted.dtu.dk, tel.: +45 4525 3650, fax: +45 45880117.

\section{THE CHARACTERISTIC EQUATION}

Figure 1 shows the general circuit which is an op amp with positive and negative feed-back. If we introduce memory elements - capacitors, coils, hysteresis - in the four admittances various types of oscillators may be obtained. The nonlinearity needed for oscillations may be introduced as nonlinear losses or hysteresis in connection with the memory elements. If we introduce linear memory elements with no hysteresis a nonlinear transfer characteristic of the amplifier must be introduced. Let us assume that the amplifier is a perfect am-

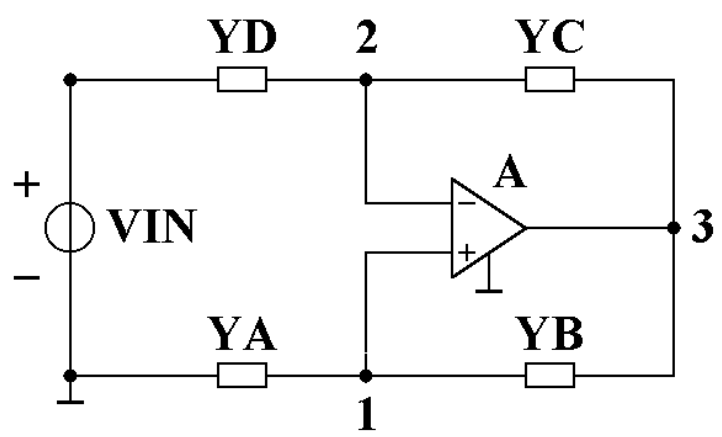

Figure 1: An amplifier with positive and negative feed-back, $V(3)=A \times V(1)-A \times V(2))$.

plifier with infinite input impedance, zero output impedance and piecewise linear gain $\mathrm{A}$. The gain is very large for small signals and zero for large signals. Now a network-function may be calculated e.g. the transfer function $V(3) / V I N$. The relation between the output voltage $V(3)$ and the input voltage $V I N$ becomes:

$$
\begin{gathered}
V(3) \times\left(\frac{Y B}{Y A+Y B}-\frac{Y C}{Y C+D}-\frac{1}{A}\right) \\
=V I N \times\left(\frac{Y D}{Y C+Y D}\right)
\end{gathered}
$$

If we observe that $V(3)$ is different from zero when $V I N$ is zero then the coefficient of $V(3)$ must be zero i.e.

$$
\left(\frac{Y B}{Y A+Y B}-\frac{Y C}{Y C+Y D}-\frac{1}{A}\right)=0
$$

Equation (2) is called the characteristic equation. The characteristic polynomial of the linearized differential equations describing the circuit 
may be derived from this equation. For infinite gain $(A=\infty)$ the equation becomes:

$$
(Y A \times Y C)-(Y B \times Y D)=0
$$

For zero gain $(A=0)$ the equation becomes:

$$
(Y A+Y B) \times(Y D+Y C)=0
$$

Table 1 is derived from the characteristic equation. It shows that it is possible to place the frequency determining circuit in any of the four admittances. If one of the admittances of Fig. 1 is chosen as a

\begin{tabular}{|c|c|c|c|}
\hline Case & Gain: & $A=0$ & $A=\infty$ \\
\hline $\mathrm{A}$ & $Y A$ & $+R B$ & $-R B \times R D / R C$ \\
\hline $\mathrm{B}$ & $Y B$ & $+R A$ & $-R A \times R C / R D$ \\
\hline $\mathrm{C}$ & $Y C$ & $+R D$ & $-R D \times R B / R A$ \\
\hline $\mathrm{D}$ & $Y D$ & $+R C$ & $-R C \times R A / R B$ \\
\hline
\end{tabular}

Table 1: The "load" of an admittance "YX" for gain zero and gain $\infty$ when the other admittances are conductors $(G X=1 / R X)$.

simple capacitor $C$ or a simple coil $L$ and the other three admittances are chosen as conductors (resistors) multi-vibrator oscillators might be designed. If one of the admittances of Fig. 1 is chosen as a simple LC parallel or series circuit and the other three admittances are chosen as conductors (resistors) negative resistance sinusoidal oscillators might be designed.

\begin{tabular}{|c|c|c|}
\hline Case: & $D$ & $A$ \\
\hline$R A$ & $180 \Omega$ & $R L C$ \\
\hline$R B$ & $180 \Omega$ & $180 \mathrm{k} \Omega$ \\
\hline$R C$ & $180 \mathrm{k} \Omega$ & $180 \Omega$ \\
\hline$R D$ & $R L C$ & $180 \Omega$ \\
\hline
\end{tabular}

Table 2: Resistor values for two cases $D$ and $A$. $R L C$ is a two-terminal with a capacitor $C=1 \mathrm{nF}$ in parallel with a coil $L=256 \mathrm{mH}$ with series resistance $R L=14.8 \Omega$.

\section{NEGATIVE RESISTANCE OSCILLA- TORS}

In [4] a $10 \mathrm{kHz}$ negative resistance oscillator is designed where $Y D$ is chosen as a coil $L_{D}=256 \mathrm{mH}$ (with a series loss resistor $R_{D}=14.8 \Omega$ ) in parallel with a capacitor $C_{D}=1 \mathrm{nF}$. The op amp $R C 4136$ is used. The components $Y A, Y B$ and $Y C$ are chosen according to case $D$ of Table 2 .

Figure 2 case $D$ shows the dynamic transfer function of the op amp. The gain $A$ is varying almost piecewise linear between zero and +2.3305 corresponding to the poles:

$$
-2.801 \mathrm{k} \pm j \times 62.44 \mathrm{k}(Q=+11.0, f=9.94 \mathrm{kHz})
$$
and

$+36.37 \mathrm{k} \pm j \times 50.78 \mathrm{k}(Q=-0.86, f=8.08 \mathrm{kHz})$ respectively in the complex frequency plane.

The....... $Q$ of a pole specified above is defined as

$$
Q=\sqrt{\left(\alpha^{2}+\omega^{2}\right)} /(-2 \times \alpha)
$$

It is a measure for the distance of the pole from the imaginary axis. It is seen that $\mathrm{Q}$ becomes $\infty$ for poles on the imaginary axis. $\mathrm{Q}$ is negative for poles in the right-half-plane RHP and positive for poles in the left-half-plane LHP. The real part of the pole may be calculated from

$$
\alpha=\omega / \sqrt{4 \times Q^{2}-1}
$$

or $2 \alpha=\omega / Q$ for large $\mathrm{Q}$.

Now the sign of the gain of the op amp is changed
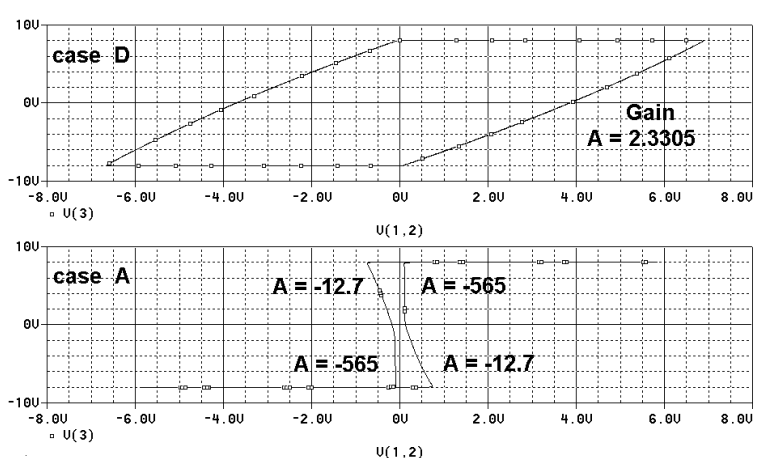

Figure 2: Op amp dynamic transfer-characteristic $V(3)$ as function of input voltage $V(1,2)$ for the two cases $D$ and $A$.

corresponding to inverting the input terminals or to interchange $Y A, Y B$ and $Y D, Y C$. This corresponds to case $A$ of Table 2 .

Figure 2 case $A$ shows the dynamic transfer function of the op amp. The gain $A$ is varying almost piecewise linear between zero, -12.7 and -565 corresponding to the poles:

$-2.801 \mathrm{k} \pm j \times 62.44 \mathrm{k}(Q=+11, f=9.94 \mathrm{kHz})$, $+1.993 \mathrm{k} \pm j \times 62.46 \mathrm{k}(Q=-16, f=9.94 \mathrm{kHz})$ and $+2.729 \mathrm{k} \pm j \times 62.43 \mathrm{k}(Q=-11, f=9.94 \mathrm{kHz})$

From Fig. 2 it is seen that both in case $D$ and in case $A$ the poles follows the whole trajectory i.e. a complex pole pair goes to the real axis and split up into two real roots. One real root goes against zero and the other real root goes to $\infty$. The two real roots meet again and a complex pole pair leaves the real axis and crosses the imaginary axis [4]. 
In case $D$ the fast transition from positive gain to zero gain via infinite gain happens at large values of the op amp input voltage $V(1,2)$. In case $A$ the fast transition from negative gain to zero gain via infinite gain happens at small values of the op amp input voltage $V(1,2)$.

Please note that it is not a jump when the real

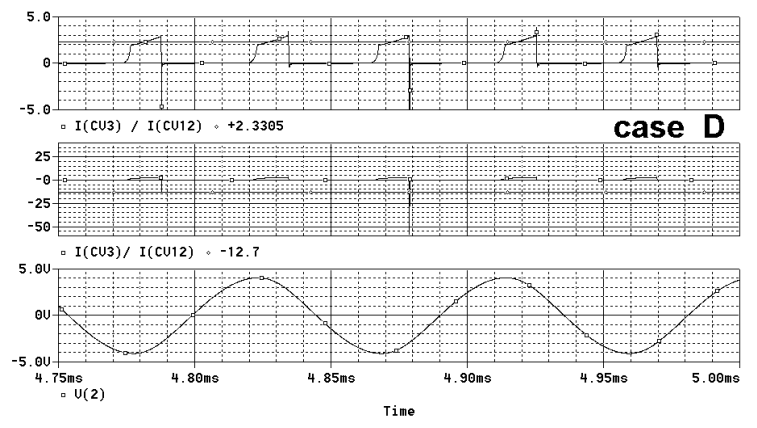

Figure 3: Gain $A=d V(3) / d V(1,2)$ and op amp inverting input $V(2)$ as functions of time, "positive gain", case $D$.

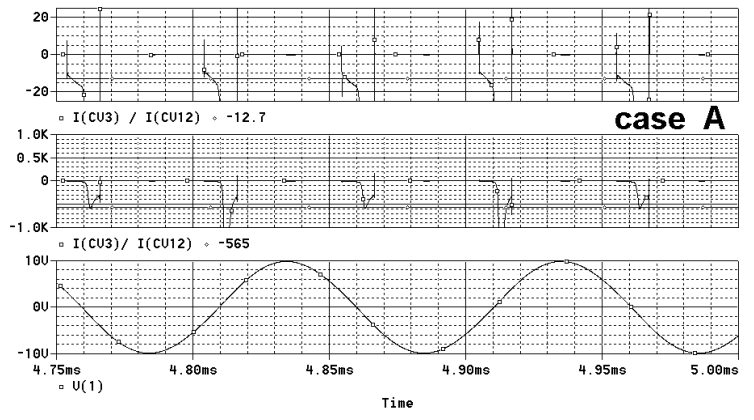

Figure 4: Gain $A=d V(3) / d V(1,2)$ and op amp non-inverting input $V(1)$ as functions of time, "negative gain", case $A$.

pole goes from $+\infty$ to $-\infty$. The Figures 3 and 4 show the gain as function of time for positive gain and negative gain respectively.

An old rule says that if your oscillator do not work then change the input terminals of the op amp. Here is given an example where the oscillator works for both positive and negative gain.

\section{A DESIGN PROCEDURE}

We want to study a negative resistance oscillator where $Y C$ is a RLC series circuit i.e. case $C$.

$$
Z C=1 / Y C=s L_{c}+R_{c}+\frac{1}{s C_{c}}
$$

with $L_{c}=256 \mathrm{mH}, R_{c}=14.8 \Omega$ and $C_{c}=1 \mathrm{nF}$.
From equation (2) we get:

$$
Y C+\left(\frac{1}{R D}\right) \times\left(\frac{\text { Num }}{\text { Denom }}\right)=0
$$

where $N u m=R B-R A \times(A-1)$

and Denom $=R A+R B \times(A+1)$

The characteristic polynomial becomes:

$$
s^{2}+2 \alpha s+\omega_{0}^{2}=0,
$$

where

$$
\begin{gathered}
2 \alpha= \\
\left(\frac{1}{L_{c}}\right) \times\left(\frac{R_{c} \times N u m+R D \times \text { Denom }}{(R B-R A \times(A-1))}\right)
\end{gathered}
$$

and

$$
\omega_{0}^{2}=\frac{1}{L_{c} \times C_{c}}
$$

$\alpha=0$ for

$$
A=\frac{(R A+R B) \times\left(R_{c}+R D\right)}{\left(R_{c} \times R A-R D \times R B\right)}
$$

Table 3 shows corresponding values of $A$ and $\alpha$.

\begin{tabular}{|c|c|}
\hline $\mathrm{A}$ & $\alpha$ \\
\hline 0 & $\left(R_{c}+R D\right) /\left(2 \times L_{c}\right)$ \\
\hline$\infty$ & $\left(R_{c} \times R A-R B \times R D\right) /\left(2 \times L_{c} \times R A\right)$ \\
\hline
\end{tabular}

Table 3: Corresponding values of $A$ and $\alpha$

The poles or the natural frequencies of the circuit - the eigenvalues of the Jacobian of the differential equations - are the roots of the characteristic polynomial.

$$
p_{1,2}=-\alpha \pm j \sqrt{\omega_{0}^{2}-\alpha^{2}}=-\alpha \pm j \omega
$$

In order to place the poles close to the imaginary axis $\alpha$ must be much smaller than $\omega_{0}$. For $Q=-10$ and $\omega=1 / \sqrt{256 m \times 1 n}=62.5 \mathrm{k}$, from equation (6) $\alpha$ becomes $3.128913589 k$. From Table $3(A=0)$ the resistor $R D$ is calculated as $R D=2 \alpha \times L_{c}-R_{c}=1.587203758 \mathrm{k} \Omega$ and from Table 1: choose $R A=R B=190 \Omega$. From equation (12) the gain is calculated as $A=-2.037649363$ which is negative. This indicates that we have to switch the input nodes of the op amp corresponding to interchange $Y A, Y B$ and $Y D, Y C$ i.e. $Y B$ becomes a RLC series circuit and we have a case $B$ oscillator instead of a case $C$ oscillator.

The complex pole pair on the imaginary axis is found as: For $A=+2.037649364$, pole: $+0.70 \mu \pm j \times 62.5 \mathrm{k}, \quad Q=-0.5 e+12 \quad$ and for $A=+2.037649363$, pole: $-64 n \pm j \times 62.5 \mathrm{k}$, 


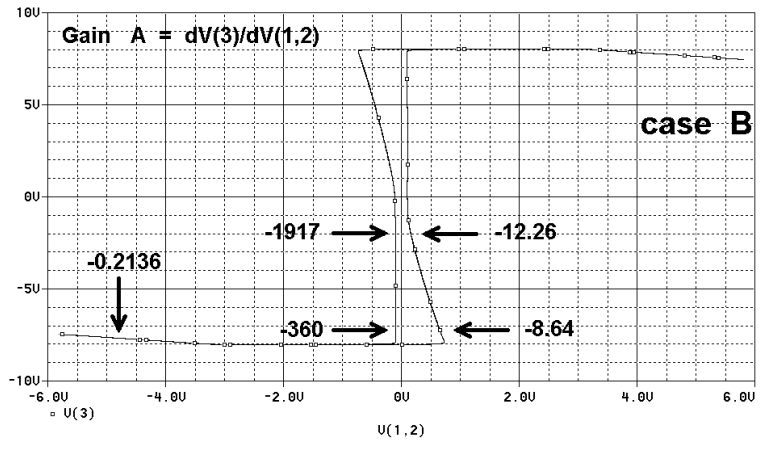

Figure 5: Op amp dynamic transfer-characteristic $V(3)$ as function of input voltage $V(2,1)$.

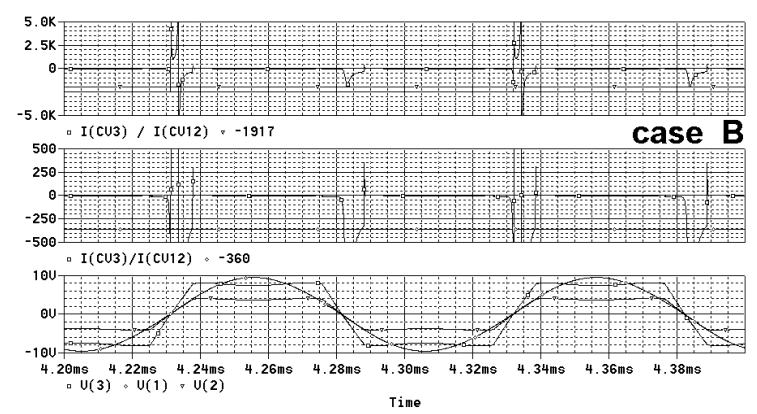

Figure 6: Gain $A=d V(3) / d V(1,2)$ as function of time.

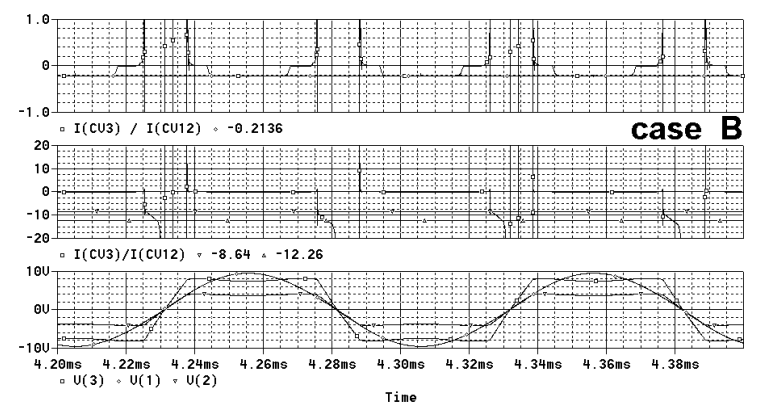

Figure 7: Gain $A=d V(3) / d V(1,2)$ as function of time.

\begin{tabular}{|c|c|c|c|}
\hline Poles & Real & Imaginary & \\
\hline Gain A & $\alpha$ & $\pm \mathrm{j} \omega$ & $\mathrm{Q}$ \\
\hline$+1 \mathrm{e}+12$ & $+3.071 \mathrm{k}$ & $62.42 \mathrm{k}$ & -10.0 \\
+38.83 & $+2.767 \mathrm{k}$ & $62.44 \mathrm{k}$ & -11.0 \\
$+1 \mathrm{e}-12$ & $-3.129 \mathrm{k}$ & $62.42 \mathrm{k}$ & +10.0 \\
$-1 \mathrm{e}-12$ & $-3.129 \mathrm{k}$ & $62.42 \mathrm{k}$ & +10.0 \\
-0.2136 & $-3.870 \mathrm{k}$ & $62.38 \mathrm{k}$ & +8.1 \\
-8.64 & $+4.939 \mathrm{k}$ & $62.30 \mathrm{k}$ & -6.3 \\
-12.26 & $+4.280 \mathrm{k}$ & $62.35 \mathrm{k}$ & -7.3 \\
-360 & $+3.106 \mathrm{k}$ & $62.42 \mathrm{k}$ & -10.0 \\
-1917 & $+3.078 \mathrm{k}$ & $62.42 \mathrm{k}$ & -10.0 \\
$-1 \mathrm{e}+12$ & $+3.071 \mathrm{k}$ & $62.42 \mathrm{k}$ & -10.0 \\
\hline
\end{tabular}

Table 4: The poles as function of the gain.
$Q=+0.5 e+12$. Figure 5 shows the op amp dynamic transfer-characteristic: $V(3)$ as function of input voltage $V(2,1)$. By zooming the characteristic the gain $A=d(V(3)) / d(V(1,2))$ is found at some places. The Figures 6 and 7 show the gain as function of time. It is interesting to observe that for rising values of $V(3)$ the infinite gain point $\infty$ is passed 4 times whereas for falling values of $V(3)$ the infinite gain point $\infty$ is passed only twice. Table 4 shows the poles for the selected values of the gain. When the gain $A$ is in the interval from -2.20865 to -1.81090 the poles are real. For $A=-2$ there are no poles. The dc-gain is found to +38.83 which corresponds to a complex pole-pair in the RHP.

Here the resistors $R D$ and $R C$ are chosen arbitrarily equal to $190 \Omega$. If we choose $R D=1900 \Omega$ and $R C=19 \Omega$ so that $R D$ and $R A=1587 \Omega$ are of the same size and so that $R C$ is of the same size as the resonance impedance of the series resonance circuit $R_{b}=14.8 \Omega$ an investigation of the spectrum by means of PSpice FFT indicates that the content of higher harmonics disappear i.e. .. . . . . .

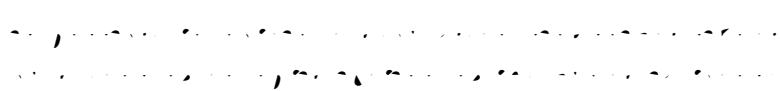

..... Also the impact of the choice of $Q$ should be further investigated.

The results depends on the power supply voltage and the type of op amp. Besides RC4136 the op amps LF411, LM324 and uA741 have been investigated with similar results.

\section{CONCLUSIONS}

Insight in the mechanisms behind the behavior of oscillators based on operational amplifiers is obtained by means of the .......... approach [3]. The dynamic transfer characteristic of a specific operational amplifier (RC4136) is studied in connection with negative resistance oscillators. A design procedure is presented.

\section{References}

[1] H. Barkhausen, Lehrbuch der ElektronenRohre, 3.Band, "Rückkopplung" , Verlag S. Hirzel, 1935.

[2] A.S. Sedra and K.C. Smith, Microelectronic Circuits 4th Ed, Oxford University Press, 1998.

[3] Erik Lindberg, "Is the Quadrature Oscillator a Multivibrator ?", IEEE Circuits \& Devices Magazine, November/December 2004, vol. 20, no. 6, pp. 23-28, 2004.

[4] Erik Lindberg, "Oscillators .. ........", (tutorial presented at ECCTD'03 - http://ecctd03.zet.agh.edu.pl/) 